FRI0447

THE SURVIVAL OF BIOLOGICS IN PSORIATIC ARTHRITIS - THE FIRST DATA FROM MOSCOW UNIFIED REGISTER OF ARTHRITIS (MUAR)

Karine Lytkina ${ }^{1}$, Evgeniya Shmidt ${ }^{2}$, Galina Lukina ${ }^{3}$, Ekaterina Koltsova ${ }^{4}$, Evgeniy Zhilyaev ${ }^{5} .{ }^{1}$ City Clinical Hospital\#4, Rheumatology department, Moscow, Russian Federation; ${ }^{2}$ City Clinical Hospital 1 named after N.I. Pirogov, Rheumatology, Moscow, Russian Federation; ${ }^{3}$ Moscow Clinical Scientific Center named after Loginov A. S., Rheumatology, Moscow, Russian Federation; ${ }^{4}$ Rheumatology Research Institute of the Organization of health and healthcare management, organizational-methodical Division, Moscow, Russian Federation; ${ }^{5}$ Rheumatology Federal State budget institution of additional professional education «Russian Medical Academy of continuing professional education» Ministry of health of Russia, Russia, Moscow, Russian Federation

Background: Early started therapy of psoriatic arthritis with the goal to achieve minimal disease activity can slow the joint damage progression. Data concerning the persistence of psoriatic arthritis (PsA) patients on biologics in real clinical practice are insufficient.

Objectives: to analyze the survival of biological treatment of PsA and detect possible predictors of persistence on biologics.

Methods: PsA patients from Moscow Unified Arthritis Registry (MUAR) treated with biologics were included in study. Unadjusted survival analysis was performed by the Kaplan-Mayer method. The search for independent risk predictors of the therapy discontinuation was carried out by the multivariate Cox regression.

Results: We analyzed 236 treatment episodes in 141 PsA patients enrolled in MUAR, 61 men (43\%); mean age was $50.1 \pm 11.5$ years. We used the following biologics: etanercept (71 patients), infliximab (51), adalimumab (50), ustekinumab (35), golimumab (11), cerolizumab pegol (7). In unadjusted analysis mean treatment survival was longer with etanercept, adalimumab and infliximab. As significant independent predictors of withdrawal risk (potential confounders) low education, mutilating or polyarticular form of PsA, heel pain, shoulder joints swelling and absence of neck pain at the disease onset were detected.

After the adjusting data for cofounders there was no significant difference in risk of withdrawal between different biologics. Neither the year of onset of the case of treatment nor the number of previous bDMARDs show a significant relation with the risk of drug withdrawal (Picture 1). This data show the difference between the patterns of treatment survival of rheumatoid arthritis and psoriatic arthritis

Conclusion: our analysis of PsA treatment persistence in real clinical practice detected several factors associated with withdrawal risk. These data can provide specialists with a tool for personalized treatment

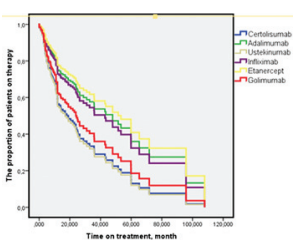

Picture 1. Adjusted analysis of persistence of bDMARDs

Disclosure of Interests: None declared

DOI: 10.1136/annrheumdis-2019-eular.4335

\section{FRI0448 $\quad$ REAL-WORLD EXPERIENCE OF SECUKINUMAB FOR PSORIATIC ARTHRITIS}

LOPEZ MARIA MARTIN ${ }^{1}$, Marta Valero ${ }^{2}$, Valentina Emperiale ${ }^{3}$, Carolina Merino Argumánez ${ }^{4}$, Javier Bachiller-Corral ${ }^{2}$, Jose Campos Esteban ${ }^{4}$, Ana Pérez Gómez ${ }^{3}$, Beatriz Joven-lbáñez ${ }^{1}{ }^{1}$ Hospital 12 de Octubre, Madrid, Spain; ${ }^{2}$ Hospital Ramón y Cajal, Madrid, Spain; ${ }^{3}$ Hospital Príncipe de Asturias, Madrid, Spain; ${ }^{4}$ Hospital Puerta de Hierro, Madrid, Spain

Background: Secukinumab, an inhibitor of IL-17, is a new option for the treatment of Psoriatic Arthritis (PsA) which has showed efficacy in clinical trials. However, real-world data of its use is still scarce.

Objectives: This study aims to analyze the experience of using secukinumab for PsA in four tertiary hospitals.

Methods: Multicentric observational, longitudinal, retrospective study conducted in 4 tertiary hospitals of the Madrid region. Patients with clinical diagnosis of PsA and having received at least one dose of secukinumab were included. Medical records were reviewed to collect demographic and clinical data (body mass index, BMI, risk factors cardiovascular disease, cancer, HBV/HCV infection), features of PsA (extra-articular involvement, radiological damage), previous therapies, assessment of the disease and response data at 6 months of secukinumab (joint counts, CRP, DAPSA, reasons for discontinuation, adverse events). Descriptive statistic analysis using mean and standard deviation was performed.

Results: 177 patients, of which 115 female (65\%) were included. Mean age was 53 y.o.(SD 15) and average duration of the disease was 9 years (SD 7). 169 patients (95\%) had peripheral disease (34\% joint erosions), $84(47 \%)$ had axial disease (68 with radiological damage), 148 $(84 \%)$ had psoriasis, 61 (34\%) showed dactilitis and $111(63 \%)$ had enthesitis. Average BMI was 28.4 (SD 6), with an obesity rate of $37 \%$ (52 pt). Observed comorbidities were hypertension (22 pt, 12\%), diabetes mellitus (22 pt, 12\%), dyslipidemia (58 pt, 33\%), active smoking (55 pt, $31 \%$ ) and others: 20 patients had HBV infection, 5 had HCV infection and $12 \%$ had malignancy.

Regarding previous treatments, $90 \%$ had received cDMARDs, particularly methotrexate $(77 \%)$ and $119(67 \%)$ had been exposed to at least one bDMARD ( $32 \%$ to one, $25 \%$ to two, $20 \%$ to three and $21 \%$ to four or more). $69 \%$ were on $150 \mathrm{mg}$ dose and $31 \%$ on $300 \mathrm{mg}$ dose.

At baseline, average tender joint count was 7 (SD 8), swollen joint count was 4 (SD 4), CRP $7 \mathrm{mg} / \mathrm{l}$ and DAPSA 26. At 6 months of secukinumab therapy, tender joint count had decreased to 5 (SD 8), swollen joint count 2 (SD 3), CRP $5 \mathrm{mg} / \mathrm{l}$ and DAPSA 17. Thirty-eight (47\%) of the patients with the data available (80) had DAPSA $\leq 14$ (low activity) and 9 DAPSA $\leq 4$ (remission). In naïve to biologic patients, DAPSA varied from 27 in the baseline visit to 16 at 6 months, and in the other group of patients with biologic experience, DAPSA varied from 24 to 17 at 6 months (if they had been exposed to 3 or more biologics, DAPSA from 18 to 10 at 6 months).

Average drug survival time was 20 months (1-34). 79 patients $(44.6 \%)$ withdrawn therapy, due to primary ineffectiveness (40), secondary ineffectiveness (26), adverse events (9) and other reasons (3). Adverse events do not differ from those reported in clinical trials.

Conclusion: Secukinumab in real-world setting had been indicated in a population of PsA with a high percentage of axial involvement (47\%), with an important previous exposure to biologics, and with higher comorbidity than that reported in clinical trials. Response data were favorable, $47 \%$ of patients achieved remission or low activity by DAPSA, and similar profile of adverse events to the one reported in the clinical trials was observed.

\section{REFERENCES:}

[1] Mease PJ, Mclnnes IB, Kirkham B, Kavanaugh A, Rahman P, van der Heijde D, et al. for the FUTURE 1 Study Group* Secukinumab Inhibition of Interleukin-17A in Patients with Psoriatic Arthritis. N Engl J Med 2015;373:1329-39

Disclosure of Interests: MARIA MARTIN LOPEZ: None declared, Marta Valero: None declared, Valentina Emperiale: None declared, Carolina Merino Argumánez: None declared, Javier Bachiller-Corral: None declared, Jose Campos Esteban: None declared, Ana Pérez Gómez: None declared, Beatriz Joven-lbáñez Speakers bureau: Celgene, Novartis, MSD, Pfizer, AbbVie, and Janssen DOI: 10.1136/annrheumdis-2019-eular.7452

\section{FRI0449 \\ EFFECTIVENESS OF BIOLOGIC DRUGS WITH DIFFERENT MECHANISM OF ACTION IN PSORIATIC ARTHRITIS. AN APPRAISAL FROM THE APULIAN REGISTRY BIOPURE}

Nicola Maruotti ${ }^{1}$, Giorgio Carlino ${ }^{2}$, Leonardo Santo ${ }^{3}$, Laura Quarta ${ }^{4}$, Romano Bucci ${ }^{5}$, Carmelo Zuccaro ${ }^{6}$, Angelo Semeraro ${ }^{7}$, Antonio Marsico ${ }^{8}$, Daniela Mazzotta ${ }^{6}$, Paola Chiara Francesca Falappone ${ }^{6}$, Florenzo lannone ${ }^{9}$

${ }^{1}$ Rheumatology Clinic, University of Foggia, Foggia, Italy; ${ }^{2}$ Rheumatology Service, ASL LE-DSS Casarano-Gallipoli (LE), Gallipoli, Italy; ${ }^{3}$ Unità Operativa di Reumatologia ASL BT, Barletta, Italy, ${ }^{4}$ U.O. of Rheumatology, "V.Fazzi" Hospital, Lecce, Lecce, Italy; ${ }^{5}$ Rheumatology Hospital Unit, A.O.U. Foggia, Foggia, Italy;

${ }^{6}$ Ambulatorio di Reumatologia Ospedale di Brindisi, Brindisi, Italy, ${ }^{7}$ Unità Operativa di Reumatologia ASL Taranto, Taranto, Italy, ${ }^{8}$ Unità Operativa di Reumatologia ASL Taranto, Taranto, Italy, ${ }^{9}$ DETO - Rheumatology Unit, University of Bari, Bari, Italy

Background: In the last decade, the number of drugs available fo patients affected with psoriatic arthritis (PsA) is increased and rheumatologists have multiple choices to manage PsA refractory to conventional dis ease modifying anti-rheumatic drugs (cDMARDs). However, the possibility 
of identifying disease's or patient's related characteristics to drive the optimal choice is still under investigation.

Objectives: We aimed at evaluating the effectiveness and drug survival of the biological drugs (bDMARDs) and apremilast indicated for the treatment of PSA patients with inadequate response to cDMARDs. To this purpose, PsA patients on treatment with bDMARs or Apremilast recorded into the Apulian BIOPURE registry have been retrospectively analyzed. Methods: We retrospectively assessed PsA patients, fulfilling CASPAR criteria, starting a biologic drug or apremilast from June 2016 through December 2017. At baseline and at last observation within the time frame of the study, DAPSA, PASI, ASDAS-CRP, the presence of enthesitis and dactylitis were collected. Rate of patients achieving DAPSA based remission was assessed at last observation. The persistence on the first treatment was evaluated by Kaplan-Meier survival curves. Estimated hazard ratios (HRs) of discontinuing therapy or achieving remission were assessed by multivariate stepwise backward Cox regression models, adjusting for patient demographics (gender, age) and disease characteristics (disease duration, number of prior bDMARDs, baseline DAPSA, PASI, presence of enthesitis and dactylitis, type of current drug).

Results: We recruited 450 PsA patients (268, 60\% female) with mean age $53( \pm 11)$ years, disease duration $19( \pm 13)$ months, $337(75 \%)$ naïve to bDMARDs. At baseline, 404 had peripheral joint involvement and 46 axial disease, DAPSA was $18( \pm 10)$, PASI $1.6( \pm 3)$, ASDAS-CRP 2.5 $( \pm 1), 48 / 355$ (13.5) had dactylitis and 78/231 (33\%) showed enthesitis. Table 1 shows the type of drugs. At last observation, the rate of patients who achieved the DAPSA remission was significantly higher in naïve patients $(25 \%)$ than in those bDMARDs experienced $(14 \%, p=0.02)$. The rate of drug survival was not significantly different among drugs (Figure 1). Cox-regression multivariate models showed that independent baseline factors negatively associated to drug persistence were prior bDMARD treatment $(\mathrm{HR} \quad 0.70,0.50-0.97(95 \% \mathrm{Cl}), \mathrm{p}=0.03)$ and the absence of axial disease ( $\mathrm{HR} 0.56,0.35-0.91(95 \% \mathrm{Cl}), \mathrm{p}=0.02)$. Negative predictors of DAPSA remission were prior bDMARD treatment (HR 0.40, 0.19-0.83 $(95 \% \mathrm{Cl}), \mathrm{p}=0.01)$, gender female (HR 0.51, 0.29-0.91 (95\% Cl), $\mathrm{p}=0.02)$, and the absence of dactylitis(HR $0.36,0.19-0.68(95 \% \mathrm{Cl}), \mathrm{p}=0.002)$.

Conclusion: A good rate of DAPSA remission is achievable with all the current available drugs in PSA in settings of real life. Predictors of remission and persistence on treatment were being male and naïve to prior bDMARDs. No difference among drugs was detected.

Table 1. Treatments

\begin{tabular}{lcc}
\hline & Frequency & Percentage \\
\hline Adalimumab & 80 & 17.8 \\
Certolizumab & 56 & 12.4 \\
Golimumab & 61 & 13.6 \\
Etanercept & 65 & 14.4 \\
Secukinumab & 104 & 23.1 \\
Ustekinumab & 43 & 9.6 \\
Apremilast & 41 & 9.1 \\
All & 450 & 100 \\
\hline
\end{tabular}

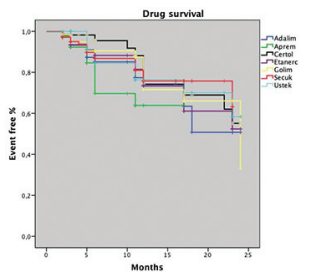

Figure 1. Kaplan-Meier curves of drug survival of PsA patients on different therapies.

Disclosure of Interests: Nicola Maruotti Speakers bureau: N Maruotti has received speaker honoraria from Pfizer outside this work, Giorgio Carlino Consultant for: G Carlino had has received consultancy fees from Pfizer, Janssen, AbbVie, MSD, BMS., Leonardo Santo Consultant for: L Santo has received consultancy fees and/or speaker honoraria from AbbVie, MSD, Novartis UCB outside this work, Speakers bureau: L Santo has received consultancy fees and/or speaker honoraria from AbbVie, MSD, Novartis UCB outside this work, Laura Quarta: None declared, Romano Bucci Consultant for: $R$ Bucci has received consultancy fees and/or speaker honoraria from Pfizer, Sanofi, MSD, BMS, Speakers bureau: R Bucci has received consultancy fees and/or speaker honoraria from
Pfizer, Sanofi, MSD, BMS, Carmelo Zuccaro Consultant for: C Zuccaro has received consultancy fees and/or speaker honoraria from MSD, AbbVie, Novartis, Pfizer, Janssen outside this work, Speakers bureau: C Zuc caro has received consultancy fees and/or speaker honoraria from MSD, AbbVie, Novartis, Pfizer, Janssen outside this work, Angelo Semeraro Speakers bureau: A Semeraro has received speaker honoraria from Sanofi, Roche, AbbVie, BMS, MSD, Novartis, Antonio Marsico: None declared, Daniela Mazzotta: None declared, Paola Chiara Francesca Falappone Consultant for: PC Falappone had received consultancy fees and/or speaker honoraria from Amgen, Abbott, MSD, BMS, outside this work, Speakers bureau: PC Falappone had received consultancy fees and/or speaker honoraria from Amgen, Abbott, MSD, BMS, outside this work, Florenzo lannone Consultant for: $F$ lannone has received consultancy fees and/or speaker honoraria from Pfizer, AbbVie, MSD, BMS Novartis, Lilly, UCB outside this work, Speakers bureau: F lannone has received consultancy fees and/or speaker honoraria from Pfizer, AbbVie, MSD, BMS, Novartis, Lilly, UCB outside this work

DOI: 10.1136/annrheumdis-2019-eular.5077

\section{FRI0450 \\ PROBABILITY OF ACHIEVING LOW DISEASE ACTIVITY OR REMISSION IN SUBJECTS WITH ACTIVE PSORIATIC ARTHRITIS TREATED WITH APREMILAST}

Philip J. Mease ${ }^{1}$, Frank Behrens ${ }^{2}$, Dafna D. Gladman ${ }^{3}$, Arthur Kavanaugh ${ }^{4}$ Michele Brunori ${ }^{5}$, Lichen Teng ${ }^{5}$, Benoit Guerette ${ }^{5}$, Ruben Queiro ${ }^{6}$, Alexis Ogdie ${ }^{7}$ ${ }^{1}$ Swedish Medical Center and University of Washington School of Medicine, Seattle, United States of America; ${ }^{2}$ Division of Rheumatology, Goethe University and Fraunhofer IME-TMP, Frankfurt, Germany; ${ }^{3} \mathrm{Krembil}$ Research Institute, Toronto Western Hospital, Toronto, Canada; ${ }^{4}$ University of California, San Diego, School of Medicine, La Jolla, United States of America; ${ }^{5}$ Celgene Corporation, Summit, United States of America; ${ }^{6}$ Rheum-Derm Unit, Hospital Universitario Central de Asturias, Oviedo, Spain; ${ }^{7}$ Perelman School of Medicine, University of Pennsylvania, Philadelphia, United States of America

Background: Therapeutic targets for long-term control of psoriatic arthritis (PsA) include the achievement of remission (REM) or low disease activity (LDA), as measured by the Clinical Disease Activity Index for Psoriatic Arthritis (CDAPSA). ${ }^{1}$

Objectives: This post hoc analysis was conducted to (1) assess the predictive values of baseline clinical disease status on achieving long-term Clinical Disease Activity for Psoriatic Arthritis (cDAPSA) targets at Week 52 and (2) examine the association between early response to APR at Week 16 and the achievement of cDAPSA targets at Week 52.

Methods: Pooled analyses of 3 phase III trials (PALACE 1-3) were performed among subjects assigned to receive APR $30 \mathrm{mg}$ twice daily at baseline. Data were analyzed using multiple imputation to account for subjects who discontinued or had missing values, using all available cDAPSA scores. The probabilities of shifting across different cDAPSA categories from baseline or Week 16 responses to Week 52 were calculated within these subjects. Binary logistic regression was also performed to confirm the results. We also analyzed mean CDAPSA from baseline to Week 52 by cDAPSA category at Week 52 among subjects with moderate or high disease activity (HDA) at baseline.

Results: A total of 494 subjects who received APR were included in the analyses; at baseline, $74.3 \%$ were in HDA, $24.5 \%$ in moderate disease activity, and $1.2 \%$ in LDA. Most subjects had $\geq 1$ of the following conditions at baseline: affected body surface area $\geq 3 \%$, dactylitis $>0$ based on the dactylitis severity score, enthesitis $>0$ based on the Maastricht Ankylosing Spondylitis Enthesitis Score or pre-existing axial PsA (physician determined) (HDA: 93.5\%; moderate disease activity: 82.6\%; LDA: 100\%). The estimated probabilities of achieving either CDAPSA LDA or REM at Week 52 were $46.9 \%$ in subjects with baseline moderate disease activity (cDAPSA $>13$ to $\leq 27$ ) and $71.1 \%$ in subjects with baseline LDA (cDAPSA $>4$ to $\leq 13$ ) (Figure). In subjects with baseline HDA (cDAPSA >27), the probability of achieving LDA or REM by Week 52 was $24.9 \%$. Subjects with moderate disease activity at baseline and improved to LDA or REM by Week 16 had high probabilities $(58.9 \%$ and $88.5 \%)$ of remaining in target at Week 52. Among the subjects with moderate disease activity at baseline, a mean cDAPSA improvement of $\geq 30 \%$ by Week 16 was associated with achievement of LDA or REM at Week 52 .

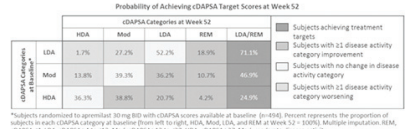

